\title{
Acompanhamento de consulta de crescimento e desenvolvimento infantil (CD) com abordagem multiprofissional - relato de experiência
}

Maria Rita Lima Lopes*, Patrícia Alves Paiva*, Gabriela Crusoé Lopes Leite de Souza*, Genesco Leonardo de Souza Nunes**, Christina Mameluque Lúcio**, Carlos Alberto Quintão Rodriques***, Danilo Cangussu Mendes****

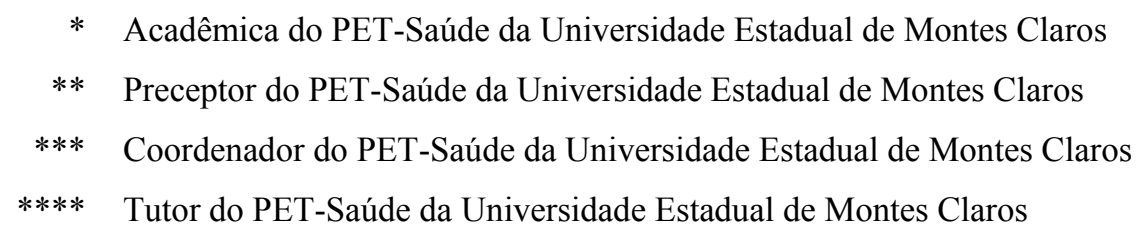

\section{RESUMO}

A consulta de acompanhamento do crescimento e desenvolvimento infantil ou puericultura, quando realizada no âmbito da Estratégia de Saúde da Família (ESF), funciona como uma ferramenta indispensável na construção do Sistema Único de Saúde, além de propor novas formas de relacionamento entre profissionais de saúde e as crianças. Ela tem como objetivo acompanhar a progressão das crianças de forma global, contemplando todas as áreas do saber no campo da saúde, a fim de possibilitar uma assistência integral e promover qualidade de vida. No entanto há poucos relatos na literatura à respeito da realização do $\mathrm{CD}$ de forma multiprofissional, com a inserção do cirurgião-dentista na puericultura. Nesse sentido, o objetivo desse trabalho é realizar um relato de experiência de caráter descritivo acerca de consultas multiprofissionais das consultas de CD realizadas na Unidade de Estratégia Saúde da Família Cintra, situada em Montes Claros, ao norte do Estado de Minas Gerais, Brasil.
Descritores: Saúde da família; Puericultura; Enfermagem pediátrica, Odontopediatria.

\section{INTRODUÇÃO}

No Brasil, a reconstrução do sistema de saúde a partir da implantação do Sistema Único de Saúde (SUS) procurou, através de seus princípios norteadores, reorientar as práticas de saúde, até então equivocadamente centradas da consulta médica e com destaque para os serviços de emergência ${ }^{8}$. Nesse contexto, a implantação da Estratégia de Saúde da Família (ESF) representou um passo fundamental, conciliando um conjunto de ações de promoção, proteção e recuperação da saúde dos indivíduos e da família, desde o recém-nascido ao idoso, de forma integral e contínua ${ }^{3}$. Segundo os pressupostos da estratégia, a atenção deve estar centrada na família, entendida e percebida a partir de seu ambiente físico e social, o que possibilita às equipes de Saúde da Família uma compreensão ampliada 
do processo saúde doença e da necessidade de intervenções que vão além da prática curativa $^{12}$. A equipe da ESF, atuando de forma multidisciplinar e prioritariamente com a promoção da saúde, tem, em tese, as melhores condições para promover a saúde infantil e materna, ainda que não tenha em sua composição o profissional especialista ${ }^{3}$.

Dentre os principais objetivos da assistência à saúde infantil desenvolvida nas Unidades de Saúde da Família (USF) estão a promoção e a recuperação da saúde das crianças, buscando assegurar um crescimento e desenvolvimento saudáveis na plenitude de suas potencialidades, sob o ponto de vista físico, mental e social ${ }^{1,10}$.

A ação primária de acompanhamento do crescimento e desenvolvimento é essencial para a articulação de atividades de prevenção e intervenção ${ }^{5}$. O Ministério da Saúde, em 1984, adotou o Programa de Atenção Integral à Saúde da Criança (PAISC), o qual priorizou cinco ações básicas a fim de incrementar a capacidade resolutiva dos serviços de saúde na atenção à criança, dentre estas ações está o acompanhamento do crescimento e desenvolvimento ${ }^{4}$.

Segundo o Ministério da Saúde ${ }^{10}$ o acompanhamento do crescimento e desenvolvimento faz parte da avaliação integral à saúde da criança (0 a 6 anos), sendo parte integrante da puericultura, a qual envolve a avaliação do peso, altura, desenvolvimento neuropsicomotor, vacinação e intercorrências, o estado nutricional, bem como orientações à mãe/família/cuidador sobre os cuidados com a criança (alimentação, higiene, vacinação, saúde bucal, aleitamento materno e estimulação) em todo atendimento, não deixando também de registrar todos os procedimentos no cartão da criança ${ }^{1}$. Esse conjunto de ações permite identificar aquelas crianças em maior risco de morbimortalidade, prevenindo precocemente a desnutrição e promovendo o crescimento infantil ${ }^{4}$.

Toda a equipe de saúde deve estar preparada para esse acompanhamento, identificando crianças de risco, fazendo busca ativa de crianças faltosas ao calendário de acompanhamento do crescimento e desenvolvimento, detectando e abordando adequadamente as alterações na curva de peso e no desenvolvimento neuro-psicomotor da criança. Esse seguimento prevê um calendário mínimo de consultas à criança propondo sete consultas no primeiro ano de vida, duas no segundo e uma por ano a partir do terceiro ano de vida até a criança completar seis anos de idade ${ }^{1}$.

Assim, toda criança até os seis anos deve possuir o Cartão da Criança, o qual deve ficar com a mãe e ser levado em todas as consultas para que se possa registrar a evolução do crescimento e desenvolvimento da criança. Para avaliação do crescimento são necessárias pesagens periódicas, com o peso ao nascer representando o primeiro ponto marcado no gráfico e os demais registros conforme o calendário mínimo de consultas da criança. Cada vez que a criança comparecer ao serviço para consulta, seja por doença ou por visita programada, deve-se pesar, registrar no gráfico do cartão e traçar a curva de peso. Sempre que possível, deve ser medido o comprimento ${ }^{4}$.

Tendo em vista que a ESF vem promovendo mudanças nas práticas de saúde e no modo como as equipes atuam nestas unidades, as ações têm sido organizadas a fim de proporcionar um atendimento integral aos seus pacientes, buscando, dessa forma, um estado de bem-estar. Refletir sobre esse aspecto do cuidado na atenção básica nos faz perceber que cuidar de crianças implica promover uma acolhida genero- 
sa, a escuta atentiva, o diálogo, o vínculo e a responsabilização ${ }^{1}$. Nesse contexto, os profissionais de saúde devem atuar conjuntamente e devem integrarem-se com outros profissionais na prestação de serviços preventivos, bem como estabelecer parcerias efetivas com todos os setores da comunidade. É essencial aprimorar as habilidades em comunicação e deve ressaltar que os procedimentos devem ser adaptados individualmente, segundo fatores de risco contextuais familiares e comunitários ${ }^{2}$.

Portanto, é imprescindível realizar a consulta de crescimento e desenvolvimento de forma multiprofissional, não abordando somente os critérios tradicionalmente analisados, mas também envolver os diversos aspectos que contribuem para o bem-estar geral da criança, fornecendo também uma cobertura e acompanhamento à saúde bucal, inserindo o cirurgião-dentista nas consultas de acompanhamento do crescimento e desenvolvimento das crianças adscritas à $\mathrm{ESF}^{1}$. No entanto é verificado que há escassos relatos na literatura a respeito da realização do $\mathrm{CD}$ de forma multiprofissional, que aborde os diversos campos da saúde (inclusive a saúde bucal) através da ação conjunta dos profissionais inseridos no contexto da estratégia em saúde da família.

Diante disso, de acordo com uma visão generalista, tendo a saúde primária como a organizadora dos cuidados à saúde, o Programa de Educação pelo Trabalho para a Saúde (PET-Saúde) objetiva proporcionar aos acadêmicos participantes uma vivência multiprofissional, a fim de alcançar a formação profissional de forma integral, calcada nas diversas nuances do processo saúde-doença. Tem, portanto, o objetivo de facilitar a integração do ensino, pesquisa e extensão e a inserção do acadêmico no ambiente da Estratégia Saúde da Família para que aplique o conhecimento aprendido na academia e interaja com os profissionais e com a comunidade assistida. Nesse contexto, o objetivo desse trabalho é realizar um relato de experiência de caráter descritivo acerca de consultas multiprofissionais do CD realizadas na Unidade de Estratégia Saúde da Família Cintra, situada em Montes Claros, ao norte do Estado de Minas Gerais, Brasil.

\section{MATERIAIS E MÉTODOS}

As consultas de crescimento e desenvolvimento foram realizadas semanalmente no período de Agosto de 2012 à Dezembro de 2013, sendo agendadas pelos agentes comunitários de saúde em diversos momentos, como por exemplo, nas consultas de pré-natal, pela livre demanda na unidade de saúde e através da visita domiciliar e/ou puerperal.

As consultas multiprofissionais foram realizadas pelos profissionais preceptores enfermeiro, médico e cirurgião-dentista, acompanhados de acadêmicos dos cursos de graduação em enfermagem, medicina, odontologia e serviço social, participantes do PET-Saúde. A estratégia de ensino entre preceptores e acadêmicos durante as consultas foi baseada em um dinamismo educacional, em que houve troca de experiências e informações entre as partes. Os acadêmicos tiveram a oportunidade de acompanhar as consultas e conhecer o protocolo seguido na realização das mesmas através de uma observação direta. A participação ocorreu de forma ativa através de questionamentos a respeito dos assuntos abordados, auxiliando tecnicamente na condução das consultas de $\mathrm{CD}$, realizando buscas literárias e sugestões aos preceptores para o aprimoramento do cuidado às crianças na atenção básica. 
No desenvolvimento das consultas de $C D$, iniciava-se realizando uma escuta qualificada dos relatos das mães/responsáveis a respeito do cotidiano das crianças e dos cuidados impostos a elas. Logo após, sob a supervisão dos preceptores, os acadêmicos puderam exercitar, durante as consultas, suas habilidades e auxiliaram na observação dos diversos indicadores do crescimento, sendo os mais comuns o peso, a estatura, os perímetros cefálico (PC), torácico e braquial, a erupção dentária e troca dos dentes (no âmbito da odontologia) o fechamento das fontanelas e suturas e, eventualmente, a idade óssea da criança. Foram ainda realizadas avaliações e aconselhamentos em relação à saúde geral e a nutrição das crianças adscritas.

Logo após, as mães foram orientadas pelo médico e enfermeiro e seus respectivos acadêmicos quanto ao calendário de vacinas, a nutrição e foram aconselhadas pela dentista e acadêmicos a respeito do aleitamento materno exclusivo, a importância de se ter hábitos alimentares saudáveis, desestimulando enfaticamente a ingestão de alimentos comprovadamente cariogênicos, principalmente açúcares refinados. Foram informadas ainda, a respeito da primeira dentição, do início da escovação dentária a partir da erupção dos primeiros dentes, enfatizando a necessidade de supervisão até o final da idade escolar, uso de fio dental e a troca dentária.

A cirurgiã-dentista e os acadêmicos de odontologia realizaram ainda nessa mesma consulta uma avaliação das necessidades bucais. Essa avaliação foi realizada com espátula de madeira, iluminação natural, em ambiente limpo e calmo e na presença da mãe/responsável. Logo após, os pacientes que necessitavam de tratamento foram encaminhados para o consultório odontológico propriamente dito para que entrassem em tratamento. Essa ação auxiliou na criação do vínculo e confiança do usuário com o profissional, tornando-se menos traumático para a criança seu primeiro acesso ao consultório odontológico, além de possibilitar um acompanhamento da condição bucal das crianças ao longo do tempo.

Durante as consultas, os preceptores trabalhavam a relação ensino-aprendizagem de forma contínua, buscando interação constante com os acadêmicos e incentivando a realização da puericultura em sua plenitude, compreendendo a criança em seu ambiente familiar e social, além de suas relações com o contexto socioeconômico, histórico, político e cultural em que está inserida, valorizando a prática da vigilância em saúde, que é um dos pilares da Estratégia saúde da Família.

\section{RESULTADOS}

A consulta de crescimento e desenvolvimento das crianças realizada de forma multiprofissional favoreceu a troca de experiências entre a equipe de saúde, acadêmicos do PET-Saúde e a população participante das consultas de CD. Isso possibilitou maior acesso da população aos profissionais em um mesmo momento, bem como favoreceu maior disponibilidade de informações preventivas/curativas com o intuito de melhorar a qualidade de vida das crianças.

Em relação à odontologia pôde-se perceber que a inclusão definitiva da saúde bucal nas consultas de CD contribuiu para que o primeiro contato da criança com o cirurgião-dentista fosse realizado de maneira atraumática e formação de vínculo com a mãe e com a criança. Durante as consultas houve a possibilidade de realização de uma avaliação precoce da saúde bucal das crian- 
ças, e, quando necessário, maior facilidade de inserção das mesmas no tratamento odontológico.

Além disso, as consultas de CD permitiram aos acadêmicos participantes o aprendizado mútuo entre os diversos campos do saber, contribuindo para uma formação mais generalista e humana, focada nas peculiaridades da família e nos princípios básicos do Sistema Único de Saúde.

\section{DISCUSSÃO}

O trabalho da ESF, quando assumido de forma integral e resolutiva, torna-se uma ação complexa, pois exige dos profissionais um arsenal de atributos e recursos tecnológicos bastante diversificados e complexos, além de um processo de trabalho que objetive a qualidade das ações desenvolvidas $^{5,} 6$. Esse processo se desenvolve mediante as várias atividades realizadas por profissionais da saúde que compartilham o mesmo ambiente físico e disponibilidade de recursos materiais. As ações desempenhadas pelos mesmos, embora sejam distintas, caracterizam-se por grande interdependência e complementaridade entre todos os membros da equipe de saúde ${ }^{1}$.

Embora seja eventualmente difícil estabelecer parâmetros de bom desempenho para as atividades de assistência à saúde da criança, existem suficientes evidências na literatura que afirmam que o controle regular da puericultura está associado ao diagnóstico precoce e intervenções oportunas e positivas para a saúde da criança ${ }^{11,13}$. De forma similar, a primeira consulta realizada de forma mais precoce pode antecipar intervenções oportunas, permitindo identificar, por exemplo, fatores de risco para o desmame precoce e corrigindo distorções dos cuidados iniciais com a criança ${ }^{11,14}$.
A coexistência de dois modelos distintos na atenção à saúde ainda parece ser uma realidade para a maioria das cidades de grande e médio portes no Brasil ${ }^{7,6}$. A assistência à saúde da criança e da mulher nos centros de saúde se mostra, quase sempre, fragmentada e respondendo apenas às demandas pontuais da população, tem-se ainda a falta de articulação entre os profissionais da equipe de saúde; falta de recursos humanos; deficiência nos serviços de referência e contra-referência, sobrecarga de atendimentos para o enfermeiro (a), em virtude da falta constante de médico nos locais de trabalho ${ }^{1,12}$.

Cabe então aos gestores de saúde a tarefa de romper com a estrutura já consolidada dos centros de saúde tradicionais, agregando ao rol de suas atividades ações sistemáticas de promoção da saúde de forma integrada e multiprofissional, estimulando a ampliação da $\mathrm{ESF}^{3}$.

É evidente que o cuidado no primeiro contato funciona como "porta de entrada" para o sistema de saúde e representa uma oportunidade de captação precoce das crianças para o acompanhamento. No entanto é relatado em diversos estudos que as ações de acompanhamento do crescimento não são realizadas com todas as crianças no primeiro contato com os serviços de atenção básica, contrariando o que é atualmente recomendado ${ }^{4}$.

A não realização das atividades mínimas para o acompanhamento do crescimento revela a necessidade de maior prioridade dos gestores sobre esta ação junto às políticas de saúde e educação, especialmente quando se deseja uma gestão plena de atenção básica e quando se busca a efetividade e o impacto do programa sobre o perfil epidemiológico da nutrição na infância, 
sobre a qualidade de vida e a mortalidade infantil ${ }^{4}$.

Trabalhos complexos, como os verificados na puericultura, exigem dos profissionais de saúde uma multiplicidade de tarefas de natureza distinta que habitualmente concorrem entre si sob o ponto de vista temporal. Face à multiplicidade e simultaneidade das atividades realizadas na ESF, os profissionais são submetidos a uma realidade de trabalho que exige deles intervenções distintas de acordo com as diferentes situações apresentadas. Deve-se considerar ainda que os profissional da saúde da família devem atuar de forma generalista, e conjuntamente, objetivando atender às demandas do serviço ${ }^{1,9}$.

Propõe então, uma nova dinâmica para a estruturação dos serviços de saúde, bem como para a sua relação com a comunidade e entre os diversos níveis de complexidade assistencial. Deve ser assumido um compromisso de prestar assistência universal, integral, contínua e, acima de tudo, resolutiva à população na unidade de saúde e no domicílio, sempre de acordo com suas reais necessidades, identificando os fatores de riscos aos quais ela está exposta e nele intervindo de forma apropriada ${ }^{1,14}$.

Em relação à formação acadêmica, é verificado que a complexidade do atendimento pediátrico extrapola a teoria e requer uma experiência organizada e orientada pela supervisão em função de objetivos a serem atingidos pelos alunos. $\mathrm{O}$ ensino necessita ser dinâmico, buscando técnicas adequadas ao contexto em que se realiza a consulta e que facilitem o crescimento dos alunos, se é pretendido que eles tenham participação ativa na construção do conhecimento $^{15}$.

\section{CONCLUSÕES}

A ESF vem se afirmando como processo instituinte capaz de contribuir para a mudança do modelo assistencial no SUS. É preconizado o enfoque da doença para uma modalidade de atenção que contemple a criança no seu processo de desenvolvimento e crescimento, além de propor a garantia da extensão de cobertura de atenção básica com capacidade resolutiva. Nessa perspectiva, há de se compreender que a ampliação da noção de desenvolvimento infantil, inclui a qualidade de vida e o bem-estar das crianças e suas famílias.

Nesse sentido, para realizar uma puericultura eficiente, deve-se compreender a criança em seu ambiente familiar e social, e deve-se atuar de forma generalista e conjunta com todos os profissionais de saúde

A multiprofissionalidade se faz importante nos espaços da saúde, pois implica em uma consciência de limites e de potencialidades de cada área do saber, buscando um fazer coletivo fundamentado nos princípios diretrizes do SUS. Desta forma o PETSaúde confirma sua importância ao habilitar profissionais com competência técnica e científica, capazes de compreender a importância do trabalho em equipe, bem como reorganizar o modo de fazer assistência à saúde da criança, a partir dos espaços de trabalho.

\section{REFERÊNCIAS}

1. Assis WD, Collet N, Reichert Altamira Pereira da Silva, Sá Lenilde Duarte de. Processo de trabalho da enfermeira que atua em puericultura nas unidades de saúde da família. Rev. bras. enferm. 2011 Feb [cited 2014 Jan 09]; 64(1): 38-46. 
2. Blank D. A puericultura hoje: um enfoque apoiado em evidências. J. Pediatr. (Rio J.), 2003 June: S13-S22.

3. Caldeira AP, Oliveira RM, Rodrigues OA. Qualidade da assistência maternoinfantil em diferentes modelos de Atenção Primária. Ciênc. saúde coletiva. 2010 Oct [cited 2014 Jan 09]; 15 ( Suppl 2 ): 3139-3147.

4. Carvalho MF, Lira PIC, Romani SAM, Santos IS, Veras AACA, Batista FM. Acompanhamento do crescimento em crianças menores de um ano: situação nos serviços de saúde em Pernambuco, Brasil. Cad. Saúde Pública [serial on the Internet]. 2008 Mar [cited 2014 Jan 09]; 24( 3 ): 675-685.

5. Corso ACT, Zeni LAZR, Heidrich EMC, Kleine L, Closs G, Botelho LJ, et al. Crianças pré-escolares e o impacto da assistência pública sobre seu crescimento. Revista Ciências da Saúde 1996; 15:29-46

6. Facchini LA, Piccini RX, Tomasi E, Thumé E, Silveira DS, Siqueira FV, Rodrigues MA. Desempenho do PSF no Sul e no Nordeste do Brasil: avaliação institucional e epidemiológica da atenção básica à saúde. Cien Saude Colet 2006; 11(3):669-681.

7. Goldbaum M, Gianini RJ, Novaes HMD, César CLG. Utilização de serviços de saúde em áreas cobertas pelo programa saúde da família (Qualis) no município de São Paulo. Rev Saude Publica 2005; 39(1):90-99.

8. Mendes EV. Uma agenda para a saúde. São Paulo: Hucitec; 1996.

9. Minayo MCS. O desafio do conhecimento: pesquisa qualitativa em saúde. 11a ed. São Paulo-Rio de Janeiro: Hucitec-Abrasco; 2009.
10. Ministério da Saúde (BR). Agenda de Compromissos para a Saúde Integral da Criança e Redução da Mortalidade Infantil. $2^{\mathrm{a}}$ reimpressão. Série A. Normas e Manuais Técnicos. Brasília: Ministério da Saúde; 2005.

11. Needlman RD. Growth and development. In: Behrman RE, Kliegman RM, Harbin AM. Nelson textbook of pediatrics. Philadelphia: WB Saunders Company; 1996. p. 30-72.

12. Reis EJFB, Santos FP, Campos FE, Acúrcio FA, Leite MTT, Leite MLC, Cherchiglia ML, Santos MA. Avaliação da qualidade dos serviços de saúde: notas . Cad Saude Publica 1990; 6(1):50-61.

13. Ribeiro EM. As várias abordagens da família no cenário do Programa/Estratégia de Saúde da Família (PSF). Rev Latino Am Enfermagem 2004; 12(4):658- 664.

14. Roncalli AG, Lima KC. Impacto de programas de saúde da família sobre indicadores de saúde da criança em municípios de grande porte da região Nordeste do Brasil. Cien Saude Colet 2006; 11(3):713-724.

15. Santos NP. Recurso semiotécnico para otimizar o desempenho de estudantes em consultas de puericultura: uma experiência piloto. Revista Brasileira de Educação Médica 2011, 35(1), 122-131.

\section{ABSTRACT \\ Follow up of growth and development consultation (CD) with multidisciplinary team approach - an experience report}

The follow-up of child growth and development (CD) or childcare, when performed under the Family Health Strategy (FHS) works as an indispensable tool in the 
construction of the Health System, also proposing new forms of relationships between health professionals and children. It aims to follow the progression of children globally, covering all areas of knowledge in the health field to enable comprehensive care and promote quality of life. However, there are few reports in the literature regarding the completion of the $\mathrm{CD}$ in a multidisciplinary perspective, with the insertion of the dentist in child care.
Thereby, the aim of this paper is to perform an descriptive experience report about multidisciplinary CD held in the Family Health Strategy Unit Cintra, located in Montes Claros, in the northern state of Minas Gerais, Brazil.

Descriptors: Family Health, Child Care, Pediatric Nursing, Pediatric Dentistry. 